\title{
Den beruflichen Wiedereinstieg erfolgreich meistern
}

\author{
Marianna Bodenmann-Zanettia ${ }^{a}$ Judith Naef ${ }^{b}$
}

${ }^{a}$ Dr. med., Vorstandsmitglied, Mitglied der FMH; ${ }^{b}$ Rechtsanwältin, Geschäftsführerin mws medical women switzerland

\begin{abstract}
Mit dem Projekt «Steigbügel» fördern medical women switzerland und die MedbaseGruppen den beruflichen Wiedereinstieg in die Grundversorgung. Das SIWF, die WHM sowie die SGAIM unterstützen das Projekt. Es richtet sich an Ärztinnen und Ärzte, die nach längerer familiär bedingter Auszeit den Facharzttitel Allgemeine Innere Medizin erlangen oder erste Berufserfahrungen nach dem Titelerwerb sammeln wollen.
\end{abstract}

An den Schweizer Universitäten wurden während Jahren zu wenig Ärztinnen und Ärzte ausgebildet. Nicht genug: Davon sind - laut einer kürzlich von der FMH und dem VSAO publizierten Studie - rund 10 Prozent nach wenigen Jahren nicht mehr kurativ tätig. Gemäss einer Umfrage des Verbandes der Schweizer Ärztinnen «mws - medical women switzerland» möchten jedoch insbesondere Ärztinnen gerne mit Patientinnen und Patienten arbeiten, sie wünschen eine Laufbahnberatung.

\section{Erfahrene Expertinnen und bedürfnis- orientierte Coachings}

Diesen Wunsch nimmt die mws mit dem wegweisenden Projekt «Steigbügel» auf: Es richtet sind an Ärztinnen, welche kurz vor dem Weiterbildungsabschluss in Allgemeiner Innerer Medizin stehen oder erste Erfahrungen

\section{Gesucht sind Sie!}

Gesucht sind nun also Sie: (fast) fertig weitergebildete Fachärztin oder weitergebildeter Facharzt für Allgemeine Innere Medizin in der Familienpause mit grosser Lust, (wieder) in die Grundversorgung einzusteigen. Interessentinnen und Interessenten melden sich bitte bei Marianna BodenmannZanetti, Fachärztin für Innere Medizin, begeisterte Lehrpraktikerin, selber Mutter von drei erwachsenen Kindern und Leiterin des Steigbügel-Projekts: Dr. med. Marianna Bodenmann-Zanetti, Eichstr. 4, 8620 Wetzikon, E-Mail: marianna.bodenmann[at]medbase.ch oder marianna.bodenmann[at] medicalwomen.ch. Sie freut sich auf Ihre Anfrage!

Weitere Informationen finden Sie auf

www.medicalwomen.ch sowie www.medbase.ch. in der Grundversorgung nach dem Abschluss dieses Facharzttitels erlangen möchten. Zukünftige Grundversorgerinnen, welche aus familiären Gründen während mindestens zwölf Monaten nicht berufstätig gewesen sind, haben dank des Projektpartners Medbase die Möglichkeit, ihre Weiterbildung zur Fachärztin fortzusetzen. Während eines Jahres sind sie als Praxisassistenzärztinnen zu 50 Stellenprozent in einer Medbase-Praxis tätig. Bei fast abgeschlossener Facharztausbildung lassen sich damit sechs Monate Assistenz an die Weiterbildung anrechnen. Der berufliche Wiedereinstieg wird durch speziell auf die Bedürfnisse dieser Gruppe entwickelte Einzel- und Gruppen-Coachings sowie besonders geschulte Lehrpraktikerinnen und Lehrpraktiker unterstützt. Die kantonalen Stellen, die das schon etablierte Praxisassistenz-Programm finanzieren, werden um Unterstützung gebeten, es können aber erhebliche Selbstkosten entstehen, die durch die Lehrpraxen gedeckt werden müssen.

Selbstverständlich sind auch weitere Gruppen- und Einzelpraxen ausserhalb der Medbase-Gruppe im Projekt willkommen: Neben Praxisassistenz-Erfahrung bringen sie idealerweise bereits selber geeignete Interessentinnen für die Teilnahme am Programm mit. Die kantonalen Stellen, die das etablierte PraxisassistenzProgramm finanzieren, werden von der mws über das Programm und seine Besonderheiten informiert. Es ist jedoch offen, ob kantonale Stellen die Lohnkosten der Praxisassistentinnen im üblichen Umfang übernehmen. Die Teilnahme am Programm kann für die Lehrpraxen deshalb mit beträchtlichen Kosten verbunden sein, die sie selber tragen müssen. Auf der anderen Seite können sie sich mit einem verantwortungsvollen Beitrag an die Zukunft des Gesundheitswesens profi- 
lieren sowie allenfalls zukünftige Mitarbeitende an sich binden.

\section{Schrittweise zurück in den Berufsalltag}

Die Praxisassistenz im Rahmen des Projektes Steigbügel unterscheidet sich somit weder in den Inhalten noch in den Zielen von den üblichen bezahlten Praxisassistenzen, sondern einzig im Weg: Die Erfahrung zeigt, dass es nach einer familiär bedingten Auszeit von auch nur schon zwei oder drei Jahren oft besonderer Anstrengungen und spezifischer Unterstützung bedarf, um im Beruf erneut erfolgreich Fuss zu fassen. Routine und Sicherheit sind wieder aufzubauen, damit der Weg zurück in den Beruf mit Erfolg bewältigt werden kann. Dieser stellt sich heute oft nicht ein, weil die Anforderungen an die Praxisassistentinnen nach einer familiären Pause manchmal zu rasch zu hoch sind. Häufig wird erwartet, dass sie ihr Wissen ab dem ersten Arbeitstag wieder vollständig abrufen und die Familie gedanklich komplett ausschalten können. Es wird ihnen also keine Chance gegeben, sich wieder im Berufsalltag einzuleben und gleichzeitig zu lernen, daneben den Familienalltag zu organisieren und zu bewältigen. Diese grosse Herausforderung zu meistern ist mit Sicherheit wesentlich öfter von Erfolg gekrönt, wenn alle Beteiligten auf diese Umstände Rücksicht zu nehmen wissen, ohne allerdings das Ziel aus den Augen zu verlieren: Nach dem «SteigbügelJahr» sollen die Ärztinnen und Ärzte wieder fest im Sattel sitzen, den weiteren Berufsweg in der Grundversorgung beherzt angehen und erfolgreich die Zügel in die Hand nehmen.

\section{Projektstart ab März 2018}

Das Projekt «Steigbügel» ist vom März 2018 bis Februar 2020 auf drei Jahre ausgelegt und erfolgt in fünf $\mathrm{Zy}$ klen mit je maximal acht Teilnehmerinnen - auch Teilnehmer sind willkommen, wenn sie die Voraussetzungen erfüllen. Diese werden während zwölf Monaten in einem «1:1»-On-the-job-Training von spezifisch geschulten Lehrpraktikerinnen und Lehrpraktikern begleitet. Mit steigender Erfahrung und Sicherheit arbeiten die Teilnehmerinnen und Teilnehmer zunehmend selbständig, gleich wie im Praxisassistenzprogramm der Stiftung zur Förderung der Weiterbildung in Hausarztmedizin WHM. Sie besuchen deren Schulungen und Netzwerkanlässe sowie praxisinterne Qualitätszirkel und Fortbildungen, zusätzlich wird der Wieder- einstieg durch das spezifische Einzel- und Gruppencoaching unterstützt.

\section{Breite Unterstützung}

Neben dem Projektpartner Medbase unterstützen das Schweizerische Institut für Weiter- und Fortbildung SIWF, die Schweizerische Gesellschaft für Allgemeine Innere Medizin SGAIM und die Stiftung zur Förderung der Weiterbildung in Hausarztmedizin WHM dieses Pilotprojekt. Das Institut für medizinische Lehre der Medizinischen Fakultät der Universität Bern (IML) führt die Evaluation durch, mit der die Erfolgsfaktoren identifiziert und konkrete Empfehlungen erarbeitet werden sollen, um die Erkenntnisse des Steigbügel-Projekts auch auf andere Fachgebiete, Sprachregionen und Unternehmen übertragen zu können. Das Projekt wird vom Eidg. Staatssekretariat für Wirtschaft (SECO) im Rahmen der Fachkräfteinitiative mitfinanziert und vom Eidg. Büro für die Gleichstellung von Frau und Mann (EBG) mit Finanzhilfen nach dem Gleichstel-

\section{Das Projekt «Steigbügel» bezweckt zudem,} für das Gesundheitswesen verloren geglaubte Ärztinnen und Ärzte zurückzugewinnen.

lungsgesetz unterstützt. Das Projekt «Steigbügel» bezweckt zudem, für das Gesundheitswesen verloren geglaubte Ärztinnen und Ärzte zurückzugewinnen. Es legt deshalb nicht nur auf die direkte Unterstützung der Teilnehmerinnen und Teilnehmer ein besonderes Augenmerk, sondern auch auf die Sensibilisierung und spezifische Weiterbildung der Lehrpraktikerinnen und Lehrpraktiker. Selbstverständlich wird es wissenschaftlich evaluiert mit dem Ziel, die Erfahrungen allgemein nutzbar zu machen und auch den Wiedereinstieg von Ärztinnen und Ärzten in Spezialfächer zu fördern.

\section{Volkswirtschaftlicher Nutzen}

Auch aus volkswirtschaftlicher Sicht ist der Wiedereinstieg von Ärztinnen und Ärzten unbedingt zu fördern: Rund 1 Mio. Franken investiert die Allgemeinheit in jede Ärztin und jeden Arzt für die über zehn Jahre dauernde Aus- und Weiterbildung bis zum Facharztdiplom. Im Vergleich dazu sind die Kosten für Coaching und Lehrpraktizierende mit 40000 Franken vergleichsweise gering. 